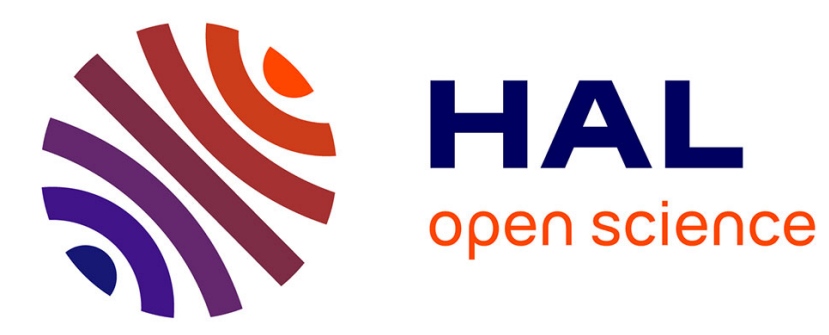

\title{
Well-posedness for a fourth order nonlinear equation related to image processing
}

Lihua Min, Xiaoping Yang, Dong Ye

\section{To cite this version:}

Lihua Min, Xiaoping Yang, Dong Ye. Well-posedness for a fourth order nonlinear equation related to image processing. Nonlinear Analysis: Real World Applications, 2014, 17, pp.192 - 202. 10.1016/j.nonrwa.2013.11.005 . hal-01095027

\section{HAL Id: hal-01095027 \\ https://hal.science/hal-01095027}

Submitted on 14 Dec 2014

HAL is a multi-disciplinary open access archive for the deposit and dissemination of scientific research documents, whether they are published or not. The documents may come from teaching and research institutions in France or abroad, or from public or private research centers.
L'archive ouverte pluridisciplinaire HAL, est destinée au dépôt et à la diffusion de documents scientifiques de niveau recherche, publiés ou non, émanant des établissements d'enseignement et de recherche français ou étrangers, des laboratoires publics ou privés. 


\title{
Well-posedness for a fourth order nonlinear equation related to image processing
}

\author{
Lihua Min $^{a, *}$ Xiaoping Yang ${ }^{b, \dagger}$ Dong Ye $^{c, \ddagger}$ \\ ${ }^{a}$ School of Science, Nanjing University of Posts and Telecommunications, \\ Nanjing 210046, Jiangsu, P.R.China \\ ${ }^{b}$ School of Science, Nanjing University of Science and Technology, \\ Nanjing 210094, Jiangsu, P.R.China \\ ${ }^{c}$ Département de Mathématiques, IECL UMR 7502, Université de Lorraine, \\ Ile du Saulcy, 57045 Metz, France
}

\begin{abstract}
Using the entropy estimates in [14], we establish the global existence and uniqueness of solutions to a fourth order equation related to image processing. Some numerical results on the Lena image are also presented to show the effectiveness of the equation for noise removal.
\end{abstract}

Keywords: fourth order parabolic equation; global existence and uniqueness; entropy estimates; numerical simulation.

AMS subject classification (2010): $35 \mathrm{~K} 35,35 \mathrm{~B} 45,35 \mathrm{~A} 01,35 \mathrm{Q} 68$.

\section{Introduction}

We study here the following fourth order initial boundary value problem:

$$
\begin{cases}u_{t}+\left(g(u) u_{x x}\right)_{x x}=0, & x \in \Omega \subset \mathbb{R}, t>0, \\ u_{x}=u_{x x x}=0, & x \in \partial \Omega, t>0, \\ u(x, 0)=u_{0}(x), & x \in \Omega,\end{cases}
$$

where $g(u)=u^{-n}$ with $n>0, \Omega$ is a bounded interval and $u_{0}$ is a positive function in $H^{1}(\Omega)$. The problem can be used to denoise in image processing and it was considered in [14]. As mentioned in [14], the diffusivity function $g(u)=u^{-n}$ corresponds to the TV diffusivity in image processing if $n=1[5,18]$, while it is the BFB diffusivity if $n=2[5,11]$.

In the past decade, many nonlinear PDEs are proposed to deal with the tradeoff between noise removal and edge preservation, the fourth-order parabolic PDEs are introduced by many scholars with the hope that these equations would perform better than their second order analogues

*E-mail: lihuamin2009@gmail.com

${ }^{\dagger}$ E-mail: yangxp@njust.edu.cn

${ }^{\ddagger}$ E-mail: dong.ye@univ-lorraine.fr 
$[20,17,21,13,2,15]$. Especially, You and Kaveh in [21] proposed the following fourth order PDE associated with a second energy functional

$$
u_{t}+\triangle\left(g_{1}(\triangle u) \triangle u\right)=0
$$

for noise removal, where

$$
g_{1}(s)=\frac{1}{1+\left(\frac{s}{k}\right)^{2}}
$$

$k>0$ is the contrast parameter. The paper [2] described some reasons to consider the fourth order PDEs in image processing. Numerical experiments, for example in $[4,21,13]$ showed that fourth-order models are able to avoid the staircasing and cartoonish effects of second order PDEs while still removing noise and preserving edges.

However, theoretical analysis of these fourth-order nonlinear PDEs is much less-developed. Because such equations often do not possess the maximum principle or comparison principle, we need some different theoretic techniques in order to obtain a priori estimates and the nonnegativity or positivity of solutions. Other substantial difficulties are often due to the strong degeneracy or singularity.

Bertozzi and Greer studied in [2] the following fourth order model:

$$
u_{t}+\nabla \cdot\left(g_{1}(\triangle u) \nabla \triangle u\right)=0
$$

which was designed to simplify an image based on its curvature. They made a key change of variables to obtain the existence and uniqueness of global smooth solutions to (1.3) in dimension one. Their work relies heavily on the special structure of $g_{1}(s)$, so the idea is difficult to be applied in our case. We also refer to [12] for the existence and uniqueness of entropy solutions to a fourth-order nonlinear degenerate parabolic equation for noise removal.

In [9], Jin and Yang considered the following fourth order problem for image restoration:

$$
\begin{cases}u_{t}+\left(g_{1}\left(u_{x}\right) u_{x x x}\right)_{x}=0, & x \in \Omega \subset \mathbb{R}, t \geq 0, \\ u_{x}=u_{x x x}=0, & x \in \partial \Omega, t \geq 0, \\ u(x, 0)=u_{0}(x), & x \in \Omega .\end{cases}
$$

Applying the Schauder fixed point method, they proved the local existence of solutions $u \in$ $C\left([0, T] ; H^{2}(\Omega)\right)$ under the assumption that $\left\|u_{0}\right\|_{H^{2}(\Omega)}$ is small enough.

Two fourth order models for noise removal with fractional derivatives as an edge detection

$$
u_{t}+\triangle\left(g_{1}\left(\left|\nabla^{1-\epsilon} u\right|\right) \triangle u\right)=0
$$

and

$$
u_{t}+\nabla \cdot\left(g_{1}\left((-\triangle)^{1-\epsilon} u\right) \nabla \triangle u\right)=0
$$

were also investigated by Guidotti and Longo [8] recently. Using the theory of maximal regularity, they proved the local existence of solutions $u \in W^{1, p}\left(0, T ; L^{p}(\Omega)\right)$ to the above two equations with periodic boundary conditions.

In the previous work [14], under the assumption of the existence of classical solutions to the equation, we discussed the large time behavior of solutions to (1.1) using some entropy estimates 
via the algebraic approach. In particular, we showed that problem (1.1) possesses some "first order" entropies.

Here we investigate the well-posedness theory of problem (1.1), i.e. the global existence and uniqueness of classical solution for suitable $u_{0}$. Throughout this paper, by a classical solution $u$ in $\Omega \times(0, T)$, we mean

$$
u \in C^{4,1}(\bar{\Omega} \times(0, T)) \cap C\left([0, T), H^{1}(\Omega)\right) .
$$

Here and after, by $H^{k}(\Omega)$, we mean the classical Sobolev spaces $W^{k, 2}(\Omega)$ for all $k \geq 1$. As $g(u)=u^{-n}$, any classical solution is clearly positive. Note that constant functions are trivial solutions to (1.1) and the total mass of any solution $u$ to (1.1) is conserved in time, that is

$$
\frac{1}{|\Omega|} \int_{\Omega} u(x, t) \mathrm{d} x=\frac{1}{|\Omega|} \int_{\Omega} u_{0}(x) \mathrm{d} x:=m, \quad t>0,
$$

where $|\Omega|$ denotes the length of $\Omega$. Due to the singularity of $g(u)$ at $u=0$, as we want to find classical solutions, we consider initial data $u_{0}$ which is small perturbation near the average $m$. Indeed, we will use the entropy estimates obtained in [14] to get appropriate estimations, and we use also an approximation process to handle the initial data $u_{0} \in H^{1}(\Omega)$. The entropy estimate is widely used to handle fourth order nonlinear parabolic equations, see for example [1] for thin film and [3] for interface fluctuation modeling.

More precisely, it was shown in [14] that for suitable couple $(n, \alpha), F_{\alpha}(u)$ is dissipative for any classical solution $u$ of (1.1), i.e.

$$
F_{\alpha}(u(x, t)) \leq F_{\alpha}\left(u_{0}\right), \quad \forall t \geq 0,
$$

where

$$
F_{\alpha}(u)=\frac{2}{\alpha^{2}} \int_{\Omega}\left(u^{\frac{\alpha}{2}}\right)_{x}^{2} \mathrm{~d} x=\frac{1}{2} \int_{\Omega} u^{\alpha-2} u_{x}^{2} \mathrm{~d} x, \quad \alpha \in \mathbb{R}^{*}:=\mathbb{R} \backslash\{0\} .
$$

More precisely, it was shown numerically that for $n \geq 1.18426$ (see Fig. 1 in [14]), we can always find $\alpha \in \mathbb{R}^{*}$ such that $F_{\alpha}$ is dissipative. Define

$$
n_{0}=\inf \left\{s>0 \text { s.t. for any } n \geq s, \exists \alpha \in \mathbb{R}^{*} \text { making } F_{\alpha} \text { dissipative }\right\} .
$$

In this paper we will give a rigorous derivation for $n_{0}<\infty$ in Appendix, where we show also a rigorous upper bound estimate for $n_{0}$. Moreover, for $n=2$, it was showed in [14] that any global classical solution $u$ to (1.1) converges uniformly to the average of the initial data $u_{0}$, with an order like $t^{-\frac{1}{4}}$ for $t \rightarrow \infty$. Here we generalize this fact for any $n \geq 1.18426$.

Our main result is

Theorem 1.1. Assume that $n>n_{0}$ and $m>0$. There exist $\sigma_{0}>0$ small, $\beta_{0}, \gamma_{0}>0$ and $C_{0}>0$ such that for any positive $u_{0} \in H^{1}(\Omega)$ with $\left\|u_{0, x}\right\|_{2} \leq \sigma_{0}$ and the given average $m$, the initial-boundary value problem (1.1) admits a unique global classical solution in the following sense:

$$
u \in C_{l o c}^{4,1}(\bar{\Omega} \times(0, \infty)) \cap C\left(\mathbb{R}_{+}, H^{1}(\Omega)\right) .
$$

The solution $u$ satisfies $0<\beta_{0} \leq u(x, t) \leq \gamma_{0}<\infty$ in $\bar{\Omega} \times[0, \infty)$ and $\left\|u_{x}(\cdot, t)\right\|_{2} \leq C_{0}\left\|u_{0, x}\right\|_{2}$ for any $t \geq 0$.

Furthermore, for $n \geq 1.18426$, there holds $u(\cdot, t) \rightarrow m$ in $C(\bar{\Omega})$ when $t$ goes to infinity. 
Here and in the following, $n_{0}$ is defined by (1.7), and $\|\cdot\|_{p}$ denotes the standard norm in $L^{p}(\Omega)$ for $p \in[1, \infty]$. For simplicity, we introduce several notations. Define $Q_{T}:=\Omega \times(0, T)$ for $T>0$, and $Q:=Q_{\infty}$. For $k=2,3$, let

$$
\dot{H}^{k}(\Omega):=\left\{v \in H^{k}(\Omega):\left.v_{x}\right|_{\partial \Omega}=0 \text { and } \int_{\Omega} v(x) \mathrm{d} x=0\right\},
$$

with the norm $\|v\|_{\dot{H}^{k}(\Omega)}=\left\|\nabla^{k} v\right\|_{2}$. Indeed, it is easy to check that the norm $\left\|\nabla^{k} v\right\|_{2}$ is equivalent to the norm $\|v\|_{H^{k}(\Omega)}$ in $\dot{H}^{k}(\Omega)$ for $k=2,3$.

Our approach can also be used to handle the the corresponding Dirichlet boundary problem

$$
\begin{cases}u_{t}+\left(g(u) u_{x x}\right)_{x x}=0, & x \in \Omega \subset \mathbb{R}, t>0, \\ u=m^{\prime}, u_{x}=0, & x \in \partial \Omega, t>0, \\ u(x, 0)=u_{0}(x), & x \in \Omega,\end{cases}
$$

where $m^{\prime}>0$ is a given constant, $g(u)=u^{-n}$ with $n>n_{0}$, and $\Omega$ is a bounded interval.

Theorem 1.2. Suppose the initial data $u_{0}$ satisfies $u_{0}-m^{\prime} \in H_{0}^{1}(\Omega)$ and $\left\|u_{0, x}\right\|_{2} \leq \sigma$ for $\sigma>0$ small enough, then there exists a unique global classical solution u to problem (1.9). Moreover for $n \geq 1.18426$, the solution satisfies

$$
u(x, t) \rightarrow m^{\prime} \quad \text { uniformly in } x \in \Omega \text {, as } t \rightarrow \infty .
$$

The rest of the paper is organized as follows. Sec. 2 is devoted to the proof of our main results. We give some numerical results to show that the model (1.1) can be effectively applied in image processing, and we make some comparison with the second order PM equation and YK equation in Sec. 3. The detailed derivation of the existence of $n_{0}$ and its estimates is given in the Appendix. Throughout the paper, we denote by $C$ (sometimes $C_{i}$ ) some generic positive constants, which may differ from line to line.

\section{Existence and uniqueness of classical solution}

Here we prove Theorem 1.1. Our main idea is the approximation method combined with the entropy estimate.

Firstly, we have the following local existence of classical solutions to problem (1.1), provided that $u_{0}$ is more regular and $\left\|u_{0, x}\right\|_{2}$ is sufficiently small.

Theorem 2.1. Let $u_{0} \in C^{4+\mu}(\bar{\Omega}), \mu \in(0,1)$ with fixed average $m>0$. Assuming that $\left\|u_{0, x}\right\|_{2}<$ $\sigma_{1}<|\Omega|^{-\frac{1}{2}} m$, problem (1.1) admits a classical solution $u \in C_{x, t}^{4+\mu, 1+\frac{\mu}{4}}\left(\overline{\mathrm{Q}_{\delta}}\right)$ for some $\delta>0$.

Proof. Using Hölder's inequality, we have $\left|u_{0}(x)-u_{0}(y)\right|<|\Omega|^{\frac{1}{2}} \sigma_{1}$ for all $x, y \in \bar{\Omega}$. In particular, there hold

$$
\max _{x \in \bar{\Omega}} u_{0}(x)-m<|\Omega|^{\frac{1}{2}} \sigma_{1}, \quad m-\min _{x \in \bar{\Omega}} u_{0}(x)<|\Omega|^{\frac{1}{2}} \sigma_{1} .
$$

Since $0<\sigma_{1}<|\Omega|^{-\frac{1}{2}} m$, there exist two positive constants $\beta_{1}, \gamma_{1}$ depending only on $\sigma_{1}, \Omega$ and $m$ such that

$$
0<\beta_{1} \leq u_{0}(x) \leq \gamma_{1}, \quad \forall x \in \bar{\Omega} .
$$


By standard parabolic Schauder theory (see Theorem 6.3 in [6], see also [7]), problem (1.1) admits a unique classical solution $u \in C_{x, t}^{4+\mu, 1+\frac{\mu}{4}}\left(\overline{\mathrm{Q}_{\delta}}\right)$ for some $\delta>0$.

The next lemma is the key estimate for our approach.

Lemma 2.2. Given $n>n_{0}$ and $m>0$, there exist $\sigma_{2}, C_{1}>0$ such that for any classical solution of (1.1) in $Q_{T}$ with some $T>0, u_{0}$ of average $m$ and $\left\|u_{0, x}\right\|_{2} \leq \sigma_{2}<|\Omega|^{-\frac{1}{2}} m$, there holds

$$
\left\|u_{x}(\cdot, t)\right\|_{2}<C_{1}\left\|u_{0, x}\right\|_{2}, \quad \text { for all } t \in(0, T) .
$$

Proof. As $n>n_{0}$, by (1.7), we can fix $\alpha \in \mathbb{R}^{*}$ such that the estimate (1.5) holds true. Let $\left\|u_{0, x}\right\|_{2}<\sigma_{1}<|\Omega|^{-\frac{1}{2}} m$. Using (2.1), there holds

$$
F_{\alpha}\left(u_{0}\right)=\frac{1}{2} \int_{\Omega} u_{0}^{\alpha-2} u_{0, x}^{2} \mathrm{~d} x<C\left\|u_{0, x}\right\|_{2}^{2}
$$

Combining with (1.5),

$$
\int_{\Omega}\left(u^{\frac{\alpha}{2}}(x, t)\right)_{x}^{2} \mathrm{~d} x=\frac{\alpha^{2}}{2} F_{\alpha}(u(x, t)) \leq \frac{\alpha^{2}}{2} F_{\alpha}\left(u_{0}\right)<\frac{\alpha^{2} C}{2}\left\|u_{0, x}\right\|_{2}^{2} .
$$

Using (1.4), there exists $x \in \bar{\Omega}$ satisfying $u^{\frac{\alpha}{2}}(x, t)=m^{\frac{\alpha}{2}}$. Similarly as the deduction of (2.1), we get

$$
\max _{x \in \bar{\Omega}} u^{\frac{\alpha}{2}}(x, t)-m^{\frac{\alpha}{2}}<C_{2}\left\|u_{0, x}\right\|_{2}, \quad m^{\frac{\alpha}{2}}-\min _{x \in \bar{\Omega}} u^{\frac{\alpha}{2}}(x, t)<C_{2}\left\|u_{0, x}\right\|_{2} .
$$

Now fix $0<\sigma_{2}<\min \left(\frac{m^{\alpha / 2}}{2 C_{2}}, \sigma_{1}\right)$. There exist then two positive constants $\beta$, $\gamma$ independent of $T$ verifying that

$$
0<\beta \leq u(x, t) \leq \gamma \text { in } Q_{T}, \quad \text { if }\left\|u_{0, x}\right\|_{2} \leq \sigma_{2} .
$$

Hence when $\left\|u_{0, x}\right\|_{2} \leq \sigma_{2}$,

$$
\int_{\Omega} u_{x}^{2} \mathrm{~d} x \leq C F_{\alpha}(u)<C\left\|u_{0, x}\right\|_{2}^{2}, \quad \forall t \in(0, T) .
$$

So we are done.

Using the above lemma, we can get now some uniform estimates for $u$ in suitable Sobolev spaces or uniform Hölder estimates locally in $t$-variable.

Lemma 2.3. Let $T_{0}>0$. Assume $\left\|u_{0, x}\right\|_{2}<\sigma_{2}$ as above. Let $u$ be a classical solution of (1.1) in $\overline{Q_{T}}$ for some $T \in\left(0, T_{0}\right)$. Then there exists a constant $M>0$ independent of $T$ such that

$$
\|u-m\|_{L^{2}\left(0, T ; \dot{H}^{3}(\Omega)\right)} \leq M
$$

and

$$
\left|u\left(x, t_{1}\right)-u\left(x, t_{2}\right)\right| \leq M\left|t_{1}-t_{2}\right|^{1 / 8}, \quad \forall x \in \bar{\Omega}, t_{1}, t_{2} \in[0, T] .
$$

Proof. Let $v(x, t):=u(x, t)-m$, then $v$ verifies

$$
\begin{cases}v_{t}+\left(g(v+m) v_{x x}\right)_{x x}=0, & x \in \Omega, t \in(0, T], \\ v_{x}=v_{x x x}=0, & x \in \partial \Omega, t \in(0, T], \\ v(x, 0)=v_{0}(x)=u_{0}(x)-m, & x \in \Omega .\end{cases}
$$


Due to $(2.3)$, there holds

$$
g(v+m) \geq \lambda>0 \quad \text { in } Q_{T} .
$$

Multiplying the equation (2.6) by $v$ and integrating over $\Omega$, we have for any $t \in(0, T)$,

$$
\frac{1}{2} \frac{d}{d t} \int_{\Omega} v^{2} \mathrm{~d} x+\int_{\Omega} g(v+m)\left|v_{x x}\right|^{2} \mathrm{~d} x=0
$$

and so

$$
\frac{1}{2} \frac{d}{d t} \int_{\Omega} v^{2} \mathrm{~d} x+\lambda \int_{\Omega}\left|v_{x x}\right|^{2} \mathrm{~d} x \leq 0
$$

which implies readily

$$
\|v\|_{C\left([0, T], L^{2}(\Omega)\right)}+\|v\|_{L^{2}\left(0, T ; \dot{H}^{2}(\Omega)\right)} \leq C\left\|v_{0}\right\|_{2} .
$$

Similarly, multiplying the equation in $(2.6)$ by $v_{x x}$, we deduce that

$$
\frac{1}{2} \frac{d}{d t} \int_{\Omega} v_{x}^{2} \mathrm{~d} x+\int_{\Omega} g(v+m)\left|v_{x x x}\right|^{2} \mathrm{~d} x=\int_{\Omega}\left(g^{\prime}(v+m) v_{x} v_{x x}\right)_{x} v_{x x} \mathrm{~d} x .
$$

Fix $\frac{1}{2}<\epsilon<1$ and $\theta=\frac{2+\epsilon}{3}<1$, by the interpolation inequality,

$$
\left\|v_{x x}\right\|_{\infty} \leq C\|v\|_{H^{2+\epsilon}(\Omega)} \leq C\|v\|_{2}^{1-\theta}\|v\|_{H^{3}(\Omega)}^{\theta} \leq C^{\prime}\|v\|_{2}^{1-\theta}\left\|v_{x x x}\right\|_{2}^{\theta} .
$$

Recalling (2.3) and (2.8), we get then

$$
\begin{aligned}
\int_{\Omega}\left(g^{\prime}(v+m) v_{x} v_{x x}\right)_{x} v_{x x} \mathrm{~d} x & \leq\left\|g^{\prime}(v+m)\right\|_{\infty}\left\|v_{x}\right\|_{2}\left\|v_{x x}\right\|_{\infty}\left\|v_{x x x}\right\|_{2} \\
& \leq C\left\|v_{x}\right\|_{2}\left\|v_{0}\right\|_{2}^{1-\theta}\left\|v_{x x x}\right\|_{2}^{1+\theta} \\
& \leq \frac{\lambda}{2}\left\|v_{x x x}\right\|_{2}^{2}+C\left\|v_{x}\right\|_{2}^{\frac{2}{1-\theta}}\left\|v_{0}\right\|_{2}^{2} .
\end{aligned}
$$

We deduce from (2.9) that

$$
\frac{d}{d t} \int_{\Omega} v_{x}^{2} \mathrm{~d} x+\lambda \int_{\Omega}\left|v_{x x x}\right|^{2} \mathrm{~d} x \leq C\left\|v_{x}\right\|_{2}^{\frac{2}{1-\theta}}\left\|v_{0}\right\|_{2}^{2} .
$$

Using now (2.2),

$$
\|v\|_{C\left([0, T] ; H^{1}(\Omega)\right)}+\|v\|_{L^{2}\left(0, T ; \dot{H}^{3}(\Omega)\right)} \leq C\left(\left\|v_{0, x}\right\|_{2}^{\frac{1}{1-\theta}}\left\|v_{0}\right\|_{2} \sqrt{T_{0}}+\left\|v_{0, x}\right\|_{2}\right) .
$$

Back to $u$, we obtain (2.4) for large enough $M$.

Setting $h(u)=g(u) u_{x x x}+g^{\prime}(u) u_{x} u_{x x}$, we have $\|h\|_{L^{2}\left(Q_{T}\right)} \leq C\left(T_{0}\right)$ according to (2.3) and Lemma 2.3. Then except the fact that the constant $M$ depends on $T_{0}$, the proof of $(2.5)$ is completely similar to that of Lemma 2.1 in [1]. So we omit the details.

Now we are ready to prove the main result.

Proof of Theorem 1.1. We divide the proof into three steps.

Step 1: Global existence. 
Let $u_{0} \in H^{1}(\Omega)$ with the fixed average $m$ and $\left\|u_{0, x}\right\|_{2} \leq \sigma_{0} \in\left(0, \sigma_{2}\right)$ where $\sigma_{2}$ is the constant given by Lemma 2.2. Take functions $u_{0, \varepsilon} \in C^{4+\mu}(\bar{\Omega})$ with the given average $m, \mu \in(0,1)$ and which approximate $u_{0}$ in $H^{1}(\Omega)$ as $\varepsilon \rightarrow 0$. We can assume that $\left\|\left(u_{0, \varepsilon}\right)_{x}\right\|_{2}<\sigma_{2}$.

Given $T_{0}>0$, apply Theorem 2.1, the initial boundary problem

$$
\begin{cases}u_{t}+\left(g(u) u_{x x}\right)_{x x}=0, & x \in \Omega, t>0, \\ u_{x}=u_{x x x}=0, & x \in \partial \Omega, t>0, \\ u(x, 0)=u_{0, \varepsilon}(x), & x \in \Omega,\end{cases}
$$

admits locally a unique classical solution $u_{\varepsilon}(x, t) \in C^{4+\mu, 1+\frac{\mu}{4}}\left(\overline{Q_{\delta_{\varepsilon}}}\right)$ for some $0<\delta_{\varepsilon} \leq T_{0}$. Moreover, using Lemmas 2.2 and 2.3, we have

$$
\left\|u_{\varepsilon}\right\|_{C_{x, t}^{\frac{1}{2}, \frac{1}{8}}\left(\overline{Q_{\delta_{\varepsilon}}}\right)} \leq C,
$$

where the constant $C$ is independent of $\varepsilon$ and $\delta_{\varepsilon}$. These a priori estimate bounds will allow us to extend the solution $u_{\varepsilon}$ step-by-step and to obtain a classical solution of (2.11) over $Q_{T_{0}}$ for any given $T_{0}>0$. So we get a global classical solution to (2.11). Moreover, combining the estimates in Lemmas 2.2 and 2.3 , there hold $\left\|u_{\varepsilon}\right\|_{C\left(\mathbb{R}_{+}, H^{1}(\Omega)\right)} \leq C$ and

$$
\left\|u_{\varepsilon}\right\|_{C^{\frac{1}{2}, \frac{1}{8}\left(\overline{Q_{T}}\right)}}+\left\|u_{\varepsilon}-m\right\|_{L^{2}\left(0, T ; \dot{H}^{3}(\Omega)\right)} \leq M(T)<\infty, \quad \forall T>0 .
$$

By the Arzelà-Ascoli theorem, up to a subsequence, we have

$$
u_{\varepsilon} \rightarrow u \quad \text { in } C_{l o c}(\bar{Q})
$$

for some function $u$. Obviously, there holds $u \in C_{l o c}^{\frac{1}{2}, \frac{1}{8}}(\bar{Q}) \cap L_{l o c}^{2}\left(\mathbb{R}_{+}, \dot{H}^{3}(\Omega)\right)$ by (2.12). Using (2.3), (2.2) and (2.4), we obtain also

$$
\left\|u_{x}\right\|_{C\left(\mathbb{R}_{+}, L^{2}(\Omega)\right)} \leq C\left\|u_{0, x}\right\|_{2}, \quad 0<\beta \leq u \leq \gamma<\infty \text { in } \bar{Q}
$$

On the other hand, by regularity theory for uniformly parabolic equations, we can conclude that $u$ is a global classical solution to (1.1).

\section{Step 2. Uniqueness}

For any $T>0$, assume $u$ and $\widetilde{u}$ are two classical solutions of problem (1.1) in $Q_{T}$. Therefore $u$ and $\widetilde{u}$ verify

$$
\left\|u_{x}\right\|_{C\left([0, T), L^{2}(\Omega)\right)}+\left\|\widetilde{u}_{x}\right\|_{C\left([0, T), L^{2}(\Omega)\right)} \leq C\left\|u_{0, x}\right\|_{2}, \quad 0<\beta \leq u, \widetilde{u} \leq \gamma<\infty \text { in } \overline{Q_{T}} .
$$

Let $\omega=u-\widetilde{u}$. By the Sobolev embedding, for any $t \in[0, T)$,

$$
\|g(u)-g(\widetilde{u})\|_{\infty}+\left\|g^{\prime}(u)-g^{\prime}(\widetilde{u})\right\|_{\infty} \leq C\left\|\omega_{x}\right\|_{2},
$$

and

$$
\begin{cases}\omega_{t}+\left(g(u) u_{x x}-g(\widetilde{u}) \widetilde{u}_{x x}\right)_{x x}=0, & \text { in } Q_{T}, \\ \omega_{x}=\omega_{x x x}=0, & \text { on } \partial \Omega \times(0, T), \\ \omega(x, 0)=0, & \text { in } \Omega .\end{cases}
$$


Multiplying (2.14) by $\omega_{x x}$, and integrating over $\Omega$, we get

$$
\begin{aligned}
& \frac{1}{2} \frac{d}{d t} \int_{\Omega} \omega_{x}^{2} \mathrm{~d} x+\int_{\Omega} g(\widetilde{u}) \omega_{x x x}^{2} \mathrm{~d} x \\
= & \int_{\Omega}\left(g^{\prime}(u) u_{x} u_{x x}-g^{\prime}(\widetilde{u}) \widetilde{u}_{x} \widetilde{u}_{x x}\right)_{x} \omega_{x x} \mathrm{~d} x+\int_{\Omega}(g(\widetilde{u})-g(u)) u_{x x x} \omega_{x x x} \mathrm{~d} x .
\end{aligned}
$$

Applying (2.7), (2.13), Young's inequality and interpolation inequality (2.10), we deduce

$$
\begin{aligned}
\frac{1}{2} \frac{d}{d t} \int_{\Omega} \omega_{x}^{2} \mathrm{~d} x+\lambda \int_{\Omega} \omega_{x x x}^{2} \mathrm{~d} x \leq & \left|\int_{\Omega}\left(g^{\prime}(u)-g^{\prime}(\widetilde{u})\right) u_{x} u_{x x} \omega_{x x x} \mathrm{~d} x\right| \\
& +\left|\int_{\Omega} g^{\prime}(\widetilde{u}) \omega_{x} u_{x x} \omega_{x x x} \mathrm{~d} x\right| \\
& +\left|\int_{\Omega} g^{\prime}(\widetilde{u}) \widetilde{u}_{x} \omega_{x x} \omega_{x x x} \mathrm{~d} x\right| \\
& +\left|\int_{\Omega}(g(u)-g(\widetilde{u})) u_{x x x} \omega_{x x x} \mathrm{~d} x\right| \\
:= & I_{1}+I_{2}+I_{3}+I_{4} .
\end{aligned}
$$

Therefore,

$$
\begin{aligned}
& I_{1} \leq C\left\|\omega_{x}\right\|_{2}\left\|u_{x}\right\|_{2}\left\|u_{x x}\right\|_{\infty}\left\|\omega_{x x x}\right\|_{2} \leq \frac{\lambda}{8}\left\|\omega_{x x x}\right\|_{2}^{2}+C(\lambda)\left\|\omega_{x}\right\|_{2}^{2}\left\|u_{x}\right\|_{2}^{2}\left\|u_{x x}\right\|_{\infty}^{2}, \\
& I_{2} \leq C\left\|\omega_{x}\right\|_{2}\left\|u_{x x}\right\|_{\infty}\left\|\omega_{x x x}\right\|_{2} \leq \frac{\lambda}{8}\left\|\omega_{x x x}\right\|_{2}^{2}+C(\lambda)\left\|\omega_{x}\right\|_{2}^{2}\left\|u_{x x}\right\|_{\infty}^{2} \\
& I_{4} \leq C\left\|\omega_{x}\right\|_{2}\left\|u_{x x x}\right\|_{2}\left\|\omega_{x x x}\right\|_{2} \leq \frac{\lambda}{8}\left\|\omega_{x x x}\right\|_{2}^{2}+C(\lambda)\left\|\omega_{x}\right\|_{2}^{2}\left\|u_{x x x}\right\|_{2}^{2}
\end{aligned}
$$

and

$$
\begin{aligned}
I_{3} \leq C\left\|\widetilde{u}_{x}\right\|_{2}\left\|\omega_{x x}\right\|_{\infty}\left\|\omega_{x x x}\right\|_{2} & \leq C\left\|\widetilde{u}_{x}\right\|_{2}\left\|\omega_{x}\right\|_{2}^{1-\theta}\left\|\omega_{x x x}\right\|_{2}^{1+\theta} \\
& \leq \frac{\lambda}{8}\left\|\omega_{x x x}\right\|_{2}^{2}+C(\lambda)\left\|\widetilde{u}_{x}\right\|_{2}^{\frac{2}{1-\theta}}\left\|\omega_{x}\right\|_{2}^{2} .
\end{aligned}
$$

We conclude then

$$
\frac{d}{d t}\left\|\omega_{x}\right\|_{2}^{2}+\left\|\omega_{x x x}\right\|_{2}^{2} \leq C(\lambda) \eta(t)\left\|w_{x}\right\|_{2}^{2} \quad \text { in }(0, T)
$$

where

$$
\eta(t)=\left\|u_{x}\right\|_{2}^{2}\left\|u_{x x}\right\|_{\infty}^{2}+\left\|u_{x x}\right\|_{\infty}^{2}+\left\|\widetilde{u}_{x}\right\|_{2}^{\frac{2}{1-\theta}}+\left\|u_{x x x}\right\|_{2}^{2}
$$

is a nonnegative function in $L^{1}(0, T)$, because $u, \widetilde{u} \in L^{2}\left(0, T ; H^{3}(\Omega)\right)$ by $(2.4)$. As $\omega(x, 0) \equiv 0$, Gronwall's inequality yields that $\left\|\omega_{x}\right\|_{2}=0$ in $(0, T)$, that is $u=\widetilde{u}$ a.e. in $\Omega \times[0, T]$. So we obtain the uniqueness of the solution.

\section{Step 3. Large-time behavior}

The case for $n=2$ has been shown in Theorem 2.3 of [14]. We can even simplify the arguments in [14] with our uniform estimate. For the sake of completeness, we give the sketches of proof. Assume first there exists $\alpha \in \mathbb{R}^{*}$ such that $(n, \alpha)$ lies in the interior of the special 
entropy region $(\mathcal{R} 1 \cap \mathcal{R} 2) \cup \mathcal{R} 3$ given in [14], see also the Appendix below. By the proof of Theorem 2.2 (especially the equality (3.12)) in [14], we get $\varepsilon_{1}>0$ such that

$$
\frac{\mathrm{d}}{\mathrm{d} t} F_{\alpha}(u) \leq-\varepsilon_{1} \int_{\Omega} u^{\alpha-n}\left(\frac{u_{x}}{u}\right)^{2}\left(\frac{u_{x x}}{u}\right)^{2} \mathrm{~d} x=-\varepsilon_{1} \int_{\Omega} u^{\alpha-n-4} u_{x}^{2} u_{x x}^{2} \mathrm{~d} x .
$$

Replacing now $G(u)$ in [14] by $F_{\alpha}(u)$, similarly as in [14], we have

$$
\frac{\mathrm{d}}{\mathrm{dt}} F_{\alpha}(u) \leq-C F_{\alpha}(u)^{3}, \quad \text { hence } F_{\alpha}(u(x, t)) \leq\left(2 C t+F_{\alpha}\left(u_{0}\right)^{-2}\right)^{-\frac{1}{2}} \text { in }(0, \infty) .
$$

Let $h=W(u)$ with $W(s)=\frac{\sqrt{2}}{\alpha} s^{\frac{\alpha}{2}}$ in $(0, \infty)$. So for any $t \in \mathbb{R}_{+}$, there exists $x_{t} \in \bar{\Omega}$ verifying $h\left(x_{t}, t\right)=W(m)$. As $\left\|h_{x}\right\|_{2}^{2}=F_{\alpha}(u)$, we get $\|h(\cdot, t)-W(m)\|_{\infty} \leq C \sqrt{F_{\alpha}(u)}$. Since $h$ is uniformly bounded by (2.3), using the mean value theorem, we conclude that

$$
\|u-m\|_{\infty}=\left\|W^{-1}(h)-m\right\|_{\infty} \leq C\|h-W(m)\|_{\infty} \leq C^{\prime} \sqrt{F_{\alpha}(u)}, \quad \forall t \in \mathbb{R}^{*} .
$$

Obviously, (2.16) and (2.15) yield that $\|u(x, t)-m\|_{\infty} \leq C t^{-\frac{1}{4}}$ for $t \rightarrow \infty$.

It remains to verify that for any $n \geq 1.18426$, there exists $\alpha \in \mathbb{R}^{*}$ such that $(n, \alpha)$ lies in the interior of $(\mathcal{R} 1 \cap \mathcal{R} 2) \cup \mathcal{R} 3$. Indeed, by (A.7) and (A.8) below, it is clear that such $\alpha$ exist for $n>\frac{14}{11}$ but also for $n \in\left(n_{1}, n_{2}\right)$.

Proof of Theorem 1.2. Using the ideas in [14], it is easy to check that entropy estimate (1.5) also holds true if $u$ is a classical solution to problem (1.9) in the sense of (1.8). As the proof of Theorem 1.2 is totally similar to that of Theorem 1.1, we leave the details for interested readers.

\section{$3 \quad$ Numerical Results}

In this section, we show the efficiency for noise removal of the proposed model

$$
u_{t}+\triangle\left(k^{n}|u|^{-n} \triangle u\right)=0
$$

by comparing with famous PM model [16]

$$
u_{t}=\nabla \cdot\left(g_{1}(|\nabla u|) \nabla u\right)
$$

and YK model (1.2), where the constant $k$ and $n$ in (3.1) are positive parameters. For simplicity, we take $n=1$ in our model (3.1) for example. A $256 \times 256$ Lena image and a captured image distorted by a Gamma multiplicative noise with mean value 1.0 and variance 0.05 (noisy image) are used for our demonstration. All experiments are implemented with MATLAB 7.8 on a core 2 personal computer, $2.40 \mathrm{GHz}, 2 \mathrm{~GB}$ RAM.

For the evaluated experiments, we use a peak signal-to-noise ratio (PSNR), a signal-to-noise ratio (SNR) and a mean structural similarity (MSSIM) index defined in [19] to measure the overall image quality. The PSNR and SNR used here are calculated as

$$
P S N R=10 \log _{10}\left\{\frac{255 \times 255}{\frac{1}{M \times N} \sum_{i=1}^{M} \sum_{j=1}^{N}\left[I(i, j)-I^{*}(i, j)\right]^{2}}\right\}
$$


and

$$
S N R=10 \log _{10}\left\{\frac{\sum_{i=1}^{M} \sum_{j=1}^{N}[I(i, j)]^{2}}{\sum_{i=1}^{M} \sum_{j=1}^{N}\left[I(i, j)-I^{*}(i, j)\right]^{2}}\right\},
$$

where $I(i, j), I^{*}(i, j)$ and $M, N$ are the original image, the image to be evaluated and the size of the image, respectively. Obviously, the higher of all the three evaluation indexes, the better of the image.
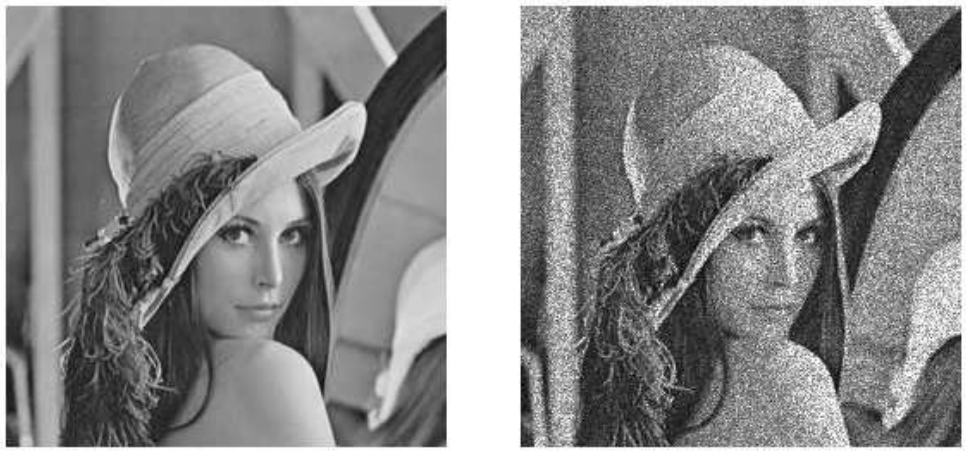

Figure 1: The original image and the noisy image
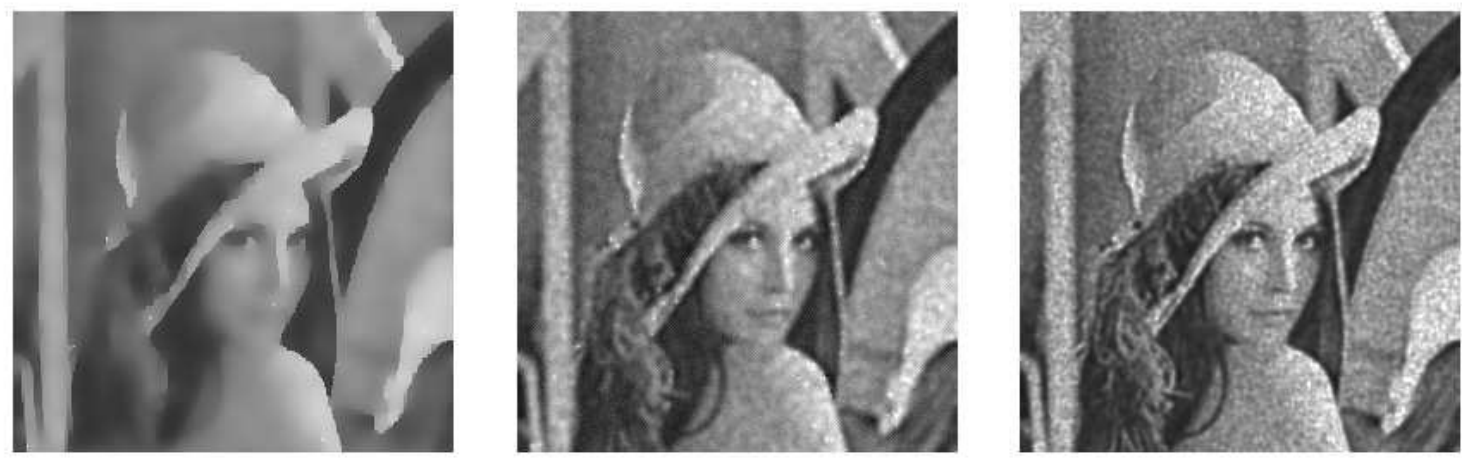

Figure 2: The restoration results by three models when $m=50$. Left: PM equation (3.2); Middle: YK equation (1.2); Right: our model (3.1).

For all the denoising models in this paper, we choose the time step size $\Delta t=0.25$ and space grid size $h=1$ in iterative approach:

$$
\begin{array}{ll}
t=m \Delta t, & m=0,1,2, \cdots \\
x=i h, & i=0,1,2, \cdots M, \\
y=j h, & j=0,1,2, \cdots N .
\end{array}
$$


Furthermore, the parameters $k$ are set to self-adaptive, 20 and 2 in (3.2), (1.2) and (3.1) respectively. Here the only parameter to tune is the number $m$ of iterations.

Fig. 1 shows the original Lena image and noisy image used in our experiments. The numerical results by using (3.1), (3.2) and YK equation (1.2) to denoise when $m=50$ are presented in Fig. 2. In Fig. 3 and Fig. 4, we compare the restoration results of the Lena image by three models if $m=100$ and $m=200$ respectively. We see from the Fig. 2-Fig. 4 that the restoration results by the proposed model are visually much better than the PM equation and YK equation.

In order to characterize the effectiveness of the model for multiplicative noise removal quantitatively, we show the PSNR, SNR and MSSIM of the noisy image and the restored image with three models when $m=50$ in Table 1, and the cases for $m=100$ and $m=200$ are presented in Table 2 and Table 3. It is clear that our model has a higher PSNR and SNR, but a lower MSSIM than the PM model for the simulated images when $m=50$ and $m=100$.
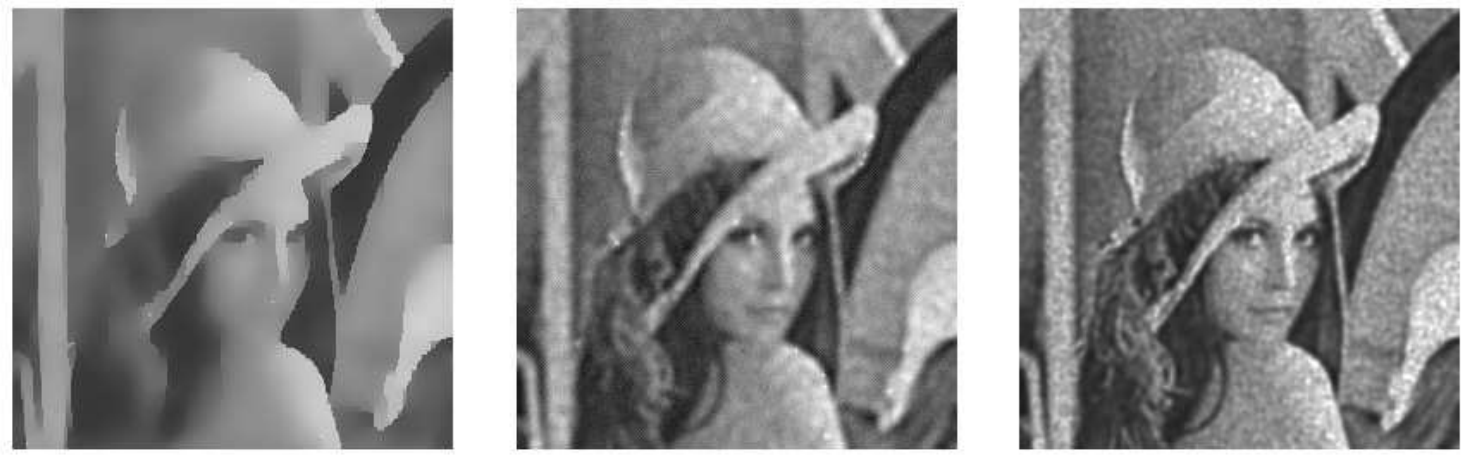

Figure 3: The restoration results by three models when $m=100$. Left: PM equation (3.2); Middle: YK equation (1.2); Right: our model (3.1).
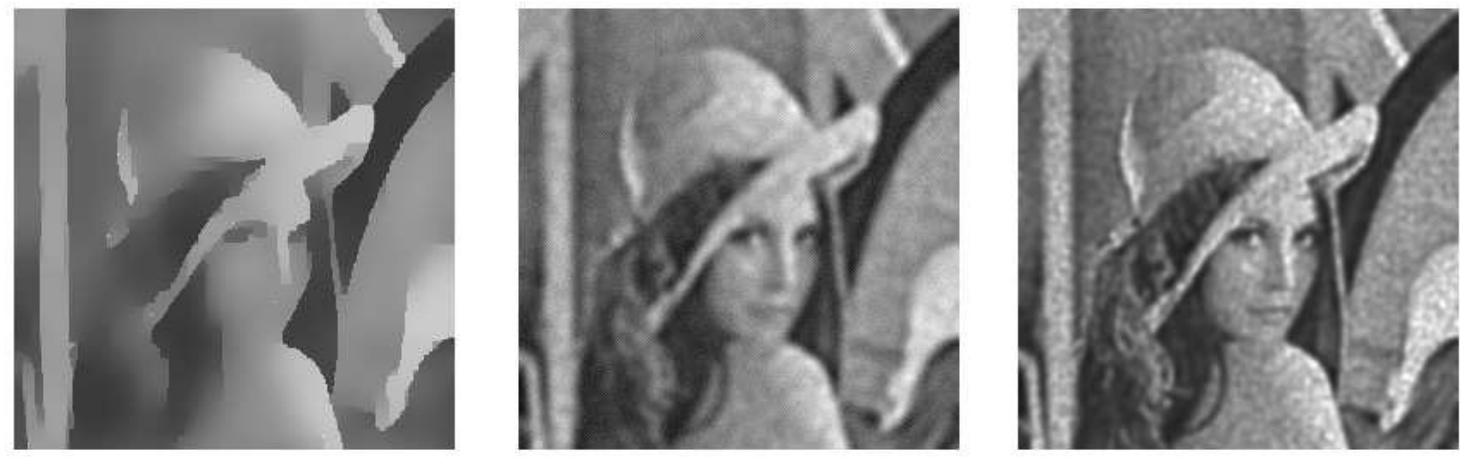

Figure 4: The restoration results by three models when $m=50$. Left: PM equation (3.2); Middle: YK equation (1.2); Right: our model (3.1). 
Table 1: The value of evaluation indexes when $m=50$

\begin{tabular}{|c|c|c|c|}
\hline Lena $\left(256^{*} 256\right)$ & PSNR & SNR & MSSIM \\
\hline noisy image & 18.6927 & 12.9683 & 0.3688 \\
\hline denoising with PM & 24.3143 & 18.5899 & 0.6985 \\
\hline denoising with YK & 24.5533 & 18.8289 & 0.5527 \\
\hline denoising with (3.1) & 25.3279 & 19.6035 & 0.6288 \\
\hline
\end{tabular}

Table 2: The value of evaluation indexes when $m=100$

\begin{tabular}{|c|c|c|c|}
\hline Lena $\left(256^{*} 256\right)$ & PSNR & SNR & MSSIM \\
\hline noisy image & 18.6927 & 12.9683 & 0.3688 \\
\hline denoising with PM & 23.5350 & 17.8106 & 0.6732 \\
\hline denoising with YK & 24.2683 & 18.5438 & 0.5407 \\
\hline denoising with $(3.1)$ & 25.7491 & 20.0247 & 0.6641 \\
\hline
\end{tabular}

Table 3: The value of evaluation indexes when $m=200$

\begin{tabular}{|c|c|c|c|}
\hline Lena $\left(256^{*} 256\right)$ & PSNR & SNR & MSSIM \\
\hline noisy image & 18.6927 & 12.9683 & 0.3688 \\
\hline denoising with PM & 22.8605 & 17.1361 & 0.6535 \\
\hline denoising with YK & 23.7784 & 18.0539 & 0.5185 \\
\hline denoising with (3.1) & 25.7147 & 19.9903 & 0.6865 \\
\hline
\end{tabular}

\section{A Derivation of $n_{0}$}

In this appendix, we give a rigorous derivation of the existence of $n_{0}$ defined by (1.7) and the structure of the following entropy region for $F_{\alpha}$ :

$$
\Sigma=\left\{(n, \alpha) \in(0, \infty) \times \mathbb{R}^{*} \text { s.t. } F_{\alpha}(u) \text { is dissipative for any classical solution to }(1.1)\right\} \text {. }
$$

In [14], the fact that $n_{0}<\infty$ was observed numerically by using $(\mathcal{R} 1 \cap \mathcal{R} 2) \cup \mathcal{R} 3 \subset \Sigma$, where

$$
\begin{aligned}
\mathcal{R} 1:= & \left\{(n, \alpha) \in(0, \infty) \times \mathbb{R}^{*} \text { s.t. }-18 \alpha^{4}-33(n-5) \alpha^{3}+\left(7 n^{2}+305 n-450\right) \alpha^{2}\right. \\
& \left.+\left(32 n^{3}+145 n^{2}-600 n+375\right) \alpha+2 n\left(6 n^{3}+5 n^{2}-100 n+125\right) \leq 0\right\} ; \\
\mathcal{R} 2:= & \left\{(n, \alpha) \in(0, \infty) \times \mathbb{R}^{*} \text { s.t. } 178 \alpha^{2}+2(92 n-335) \alpha+\left(13 n^{2}-380 n+700\right)<0\right\} ; \\
\mathcal{R} 3:= & \left\{(n, \alpha) \in(0, \infty) \times \mathbb{R}^{*} \text { s.t. } 53 \alpha^{4}+2(56 n-191) \alpha^{3}+3\left(27 n^{2}-196 n+379\right) \alpha^{2}\right. \\
& \left.+\left(28 n^{3}-231 n^{2}+1188 n-1660\right) \alpha+\frac{1}{4}\left(29 n^{4}-56 n^{3}+840 n^{2}-3680 n+3920\right) \leq 0\right\} .
\end{aligned}
$$

For completeness, we first recall some calculations for the entropy region using the following two lemmas in [10].

Lemma A.1. Let the real polynomial be

$$
P\left(\xi_{1}, \xi_{2}, \xi_{3}\right)=a_{1} \xi_{1}^{6}+a_{2} \xi_{1}^{4} \xi_{2}+a_{3} \xi_{1}^{3} \xi_{3}+a_{4} \xi_{1}^{2} \xi_{2}^{2}+a_{5} \xi_{1} \xi_{2} \xi_{3}+\xi_{3}^{2} .
$$


Then $P\left(\xi_{1}, \xi_{2}, \xi_{3}\right) \geq 0$ in $\mathbb{R}^{3}$ is equivalent to have

$$
\begin{aligned}
\text { either } & 4 a_{4}-a_{5}^{2}>0 \text { and } 4 a_{1} a_{4}-a_{1} a_{5}^{2}-a_{2}^{2}-a_{3}^{2} a_{4}+a_{2} a_{3} a_{5} \geq 0 \\
\text { or } & 4 a_{4}-a_{5}^{2}=2 a_{2}-a_{3} a_{5}=0 \text { and } 4 a_{1}-a_{3}^{2} \geq 0
\end{aligned}
$$

Lemma A.2. Let $P(x)=a_{0}+a_{1} x+a_{2} x^{2}$ with $a_{2}>0$. For any $\hat{x} \in \mathbb{R}$, then $\inf _{x>\hat{x}} P(x)<0$ is equivalent to have either $P(\hat{x})<0$ or $4 a_{0} a_{2}-a_{1}^{2} \leq 0$ and $2 a_{2} \hat{x}+a_{1}<0$.

Recall the proof of Theorem 2.2 in [14]: $F_{\alpha}$ is a dissipative entropy if there exist constants $c_{4}$ and $c_{5} \in \mathbb{R}$, making

$$
\begin{aligned}
S_{c_{4}, c_{5}}(\xi)= & c_{4}(\alpha-n-5) \xi_{1}^{6}+\left[\frac{(\alpha-2)(\alpha-3)}{2}+c_{5}\right] \xi_{1}^{3} \xi_{3} \\
& +\left[c_{5}(\alpha-n-4)+5 c_{4}-\frac{n(\alpha-2)(\alpha-3)}{2}\right] \xi_{1}^{4} \xi_{2} \\
& +\left[3 c_{5}-2 n(\alpha-2)\right] \xi_{1}^{2} \xi_{2}^{2}+(2 \alpha-4-n) \xi_{1} \xi_{2} \xi_{3}+\xi_{3}^{2}
\end{aligned}
$$

a nonnegative polynomial in $\mathbb{R}^{3}$.

Using Lemma A.1, this problem is equivalent to either

$$
0<a_{4}-a_{5}^{2}=-4 \alpha^{2}+12 c_{5}-4 \alpha(-4+n)-(-4+n)^{2},
$$

and

$$
\begin{aligned}
0 \leq p\left(c_{4}, c_{5}\right):= & 4 a_{1} a_{4}-a_{1} a_{5}^{2}-a_{2}^{2}-a_{3}^{2} a_{4}+a_{2} a_{3} a_{5} \\
= & -25 c_{4}^{2}+c_{4}\left[20+\alpha^{3}-9 n-3 n^{2}+n^{3}+\alpha^{2}\left(1+\frac{5 n}{2}\right)\right. \\
& \left.-c_{5}(40+7 n)+\alpha\left(-16+12 c_{5}-\frac{n}{2}+3 n^{2}\right)\right] \\
& +\frac{c_{5}}{4}\left\{\alpha^{4}+\alpha^{2}\left(65-8 c_{5}-18 n\right)+2 \alpha^{3}(-7+n)\right. \\
& \left.-4\left(-21+18 c_{5}+3 c_{5}^{2}+12 n+4 c_{5} n\right)+4 \alpha\left[-31+13 n+c_{5}(11+n)\right]\right\} \\
:= & A_{1} c_{4}^{2}+A_{2} c_{4}+A_{3} ;
\end{aligned}
$$

or

$$
\begin{aligned}
& 0=4 a_{4}-a_{5}^{2}=-4 \alpha^{2}+12 c_{5}-4 \alpha(-4+n)-(-4+n)^{2}, \\
& 0=2 a_{2}-a_{3} a_{5}=\frac{1}{2}\left(24-32 \alpha+14 \alpha^{2}-2 \alpha^{3}+20 c_{4}-8 c_{5}-6 n+5 \alpha n-\alpha^{2} n-2 c_{5} n\right), \\
& 0 \leq 4 a_{1}-a_{3}^{2}=-\frac{1}{4}\left(6-5 \alpha+\alpha^{2}+2 c_{5}\right)^{2}+4 c_{4}(-5+\alpha-n) .
\end{aligned}
$$

First, we solve the case (A.2)-(A.3). The inequality (A.2) gives readily

$$
c_{5}>\widetilde{c_{5}}=: \frac{1}{12}\left(16-16 \alpha+4 \alpha^{2}-8 n+4 \alpha n+n^{2}\right) .
$$


Moreover, for fixed $c_{5}, p\left(c_{4}, c_{5}\right)$ is a quadratic polynomial in $c_{4}$ with a strictly negative leading coefficient. Therefore, there exists $c_{4} \in \mathbb{R}$ such that $p\left(c_{4}, c_{5}\right) \geq 0$ if and only if $p\left(c_{4}, c_{5}\right)$ has a nonnegative discriminant: $0 \leq \Delta:=-3\left(c_{5}-\widetilde{c_{5}}\right) \Delta_{1}\left(c_{5}\right)$ where

$$
\begin{aligned}
\Delta_{1}\left(c_{5}\right)= & 100 c_{5}^{2}+\left(200-180 \alpha+52 \alpha^{2}-120 n+56 \alpha n-8 n^{2}\right) c_{5} \\
& +\left[100-60 \alpha-11 \alpha^{2}+6 \alpha^{3}+\alpha^{4}-40 n-28 \alpha n+16 \alpha^{2} n\right. \\
& \left.+4 \alpha^{3} n-36 n^{2}+20 \alpha n^{2}+8 \alpha^{2} n^{2}+8 n^{3}+8 \alpha n^{3}+4 n^{4}\right] \\
:= & a_{0}+a_{1} c_{5}+a_{2} c_{5}^{2} .
\end{aligned}
$$

So, we need only to determine those values of $\alpha$, for which there exists $c_{5}>\widetilde{c_{5}}$ making $\Delta_{1}\left(c_{5}\right) \leq 0$. By direct verifications, we can check that $4 a_{0} a_{2}-a_{1}^{2} \leq 0$ gives exactly the region $\mathcal{R} 1$, and $2 a_{2} \widetilde{c_{5}}+a_{1}<0$ gives exactly the region $\mathcal{R} 2$; while the condition $a_{0}+a_{1} \widetilde{c_{5}}+a_{2}{\widetilde{c_{5}}}^{2}<0$ corresponds exactly to the region $\mathcal{R} 3$. Applying Lemma A.2, we have already

$$
(\mathcal{R} 1 \cap \mathcal{R} 2) \cup \mathcal{R} 3 \subset \Sigma .
$$

For the case (A.4)-(A.6), we can remark that conditions (A.4) and (A.5) yield

$$
c_{5}=\frac{1}{12}\left(16-16 \alpha+4 \alpha^{2}-8 n+4 \alpha n+n^{2}\right)
$$

and

$$
c_{4}=\frac{1}{120}\left(-80+128 \alpha-68 \alpha^{2}+12 \alpha^{3}+20 n-30 \alpha n+10 \alpha^{2} n-4 n^{2}+4 \alpha n^{2}+n^{3}\right) .
$$

We can check that the inequality (A.6) implies again $(n, \alpha) \in \mathcal{R} 3$.

Therefore, we conclude that the functional $F_{\alpha}(u)$ is dissipative for any classical solution $u$ of (1.1) if the couple $(n, \alpha) \in(\mathcal{R} 2 \cap \mathcal{R} 2) \cup \mathcal{R} 3$. Now we are in position to derive a quick estimate for $n_{0}$.

Consider first the region $\mathcal{R} 1 \cap \mathcal{R} 2$. In fact, we can resolve directly the polynomial in the definition of $\mathcal{R} 1$. For any $n \in \mathbb{R}$, we have four roots:

$$
\frac{5-3 n}{2}, \frac{5-2 n}{3},-\frac{2 n}{3}, 5+n \text {. }
$$

On the other hand, we can see that $(n, \alpha) \in \mathcal{R} 2$ if and only if $n>\frac{1}{82}(\sqrt{15130}-40) \approx 1.012$, and $0 \neq \alpha \in\left(\alpha_{-}, \alpha_{+}\right)$, where $\alpha_{ \pm}$are the two roots of the polynomial in the definition of $\mathcal{R} 2$, i.e.

$$
\alpha_{ \pm}=\frac{1}{178}\left[335-92 n \pm 5 \sqrt{3\left(82 n^{2}+80 n-165\right)}\right] .
$$

To conclude, $(n, \alpha) \in \mathcal{R} 1 \cap \mathcal{R} 2 \subset \Sigma$ for $\alpha \neq 0$ verifying

$$
\alpha_{1}<\alpha \leq \frac{5-2 n}{3}, \quad \text { if } \frac{14}{11}<n \leq 6 ; \quad-\frac{2 n}{3} \leq \alpha \leq \frac{5-2 n}{3} \quad \text { if } n>6 .
$$

We get immediately that $n_{0} \leq 14 / 11 \approx 1.273$, and $\stackrel{\circ}{\Sigma} \cap^{\circ}\left(\{n\} \times \mathbb{R}^{*}\right) \neq \emptyset$ for $n>14 / 11$. 
Furthermore, with the help of the software Mathematica, we can also determine exactly the region $\mathcal{R} 3$. Indeed, let $n_{1}$ and $n_{2}$ be the second and third real roots of the polynomial

$$
1210 x^{7}-792 x^{6}-40256 x^{5}-40398 x^{4}+150211 x^{3}+27738 x^{2}-252855 x+182520 .
$$

We have $n_{1}<1.18426$ and $n_{2}>6$. For $n \in\left[n_{1}, n_{2}\right]$ fixed, let $\alpha_{1, n}$ and $\alpha_{2, n}$ be the first and second real roots of the following polynomial (we can check that $\alpha_{1, n}, \alpha_{2, n}$ exist):

$$
\begin{aligned}
& 212 x^{4}+(448 n-1528) x^{3}+\left(324 n^{2}-2352 n+4548\right) x^{2} \\
& +\left(112 n^{3}-924 n^{2}+4752 n-6640\right) x+29 n^{4}-56 n^{3}+840 n^{2}-3680 n+3920 .
\end{aligned}
$$

We get then

$$
\mathcal{R} 3=\left\{(n, \alpha) \in\left[n_{1}, n_{2}\right] \times \mathbb{R}^{*}, \alpha \in\left[\alpha_{1, n}, \alpha_{2, n}\right]\right\}
$$

We can conclude now $n_{0} \leq n_{1}$ and $\stackrel{\circ}{\Sigma}^{\prime}\left(\{n\} \times \mathbb{R}^{*}\right) \neq \emptyset$ for any $n \geq 1.18426$, as $\alpha_{1, n}<\alpha_{2, n}$ for $n \in\left(n_{1}, n_{2}\right)$.

Acknowledgement: The authors thank the anonymous reviewers for their careful reading and valuable suggestions. This work was partially completed while the first author was visiting the department of mathematics at university of Lorraine-Metz. She would like to thank its hospitality and express her deep gratitude to Professor Jean-Pierre Croisille for the help in study and life during that time. X. Yang is supported by National Natural Science Foundation of China (No. 91330101).

\section{References}

[1] F. Bernis, A. Friedman, Higher-order nonlinear degenerate parabolic equations, J. Diff. Equations, 83 (1990), 179-206.

[2] A. Bertozzi, J. Greer, Low-curvature image simplifiers: global regularity of smooth solutions and Laplacian limiting schemes, Comm. Pure Appl. Math., 57(6) (2004), 764-790.

[3] P. Bleher, J. Lebowitz, E. Speer, Existence and positivity of solutions of a fourth-order nonlinear PDE describing interface fluctuations, Comm. Pure Appl. Math., 47 (1994), 923-942.

[4] S. Didas, J. Weickert, B. Burgeth, Stability and local feature enhancement of higher order nonlinear diffusion filtering, in: W. Kropatsch, R. Sablatnig, A. Hanbury (Eds.), Pattern Recognition, Lecture Notes in Computer Science, vol. 3663, Springer, Berlin, (2005), 451-458.

[5] S. Didas, J. Weickert, B. Burgeth, Properties of higher order nonlinear diffusion filtering, J. Math. Imaging Vision, 35(3) (2009), 208-226.

[6] S. Eidel'man, Parabolic systems, Translated from the Russian by Scripta Technica, London. North-Holland Publishing Co., Amsterdam, 1969.

[7] A. Friedman, Interior estimates for parabolic systems of partial differential equations, J. Math. Mech., 7 (1958), 393-418.

[8] P. Guidotti, K. Longo, Well-posedness for a Class of Fourth Order Diffusions of Image Processing, Nonlinear Differ. Equ. Appl., 18(4) (2011), 407-425.

[9] Z. Jin, X. Yang, Strong solutions for the generalized Perona-Malik equation for image restoration, Nonlinear Anal., 73 (2010), 1077-1084.

[10] A. Jüngel, D. Matthes, An algorithmic construction of entropies in higher-order nonlinear PDEs, Nonlinearity, 19(3) (2006), 633-659. 
[11] S. Keeling, R. Stollberger, Nonlinear anisotropic diffusion filters for wide range edge sharpening, Inverse Problems, 18 (2002), 175-190.

[12] Q. Liu, Z. Yao, Y. Ke, Entropy solutions for a fourth-order nonlinear degenerate problem for noise removal, Nonlinear Anal., 67 (2007), 1908-1918.

[13] M. Lysaker, A. Lundervold, X. Tai, Noise removal using fourth-order partial differential equation with applications to medical magnetic resonance images in space and time, IEEE Trans. Image Process., 12 (2003), 1579-1590.

[14] L. Min, X. Yang, C. Gui, Entropy estimates and large time behavior of solutions to a fourth-order nonlinear degenerate equation, Commun. Contemp. Math., 15(4) (2013), 1250066, 23 pp.

[15] S. Osher, A. Solś, L. Vese, Image decomposition and restoration using total variation minimization and the $H^{-1}$ norm, Multiscale Model. Simul., 1(3) (2003), 349-370.

[16] P. Perona, J. Malik, Scale-space and edge detection using anisotropic diffusion, IEEE Trans. Pattern Anal. Machine Intell., 12 (1990), 629-639.

[17] J. Tumblin, G. Turk, LCIS: a boundary hierarchy for detail-preserving contrast reduction, In Proceedings of the SIGGRAPH 1999 Annual Conference on Computer Graphics, August 8-13, 1999, Los Angeles, CA, USA, Siggraph Annual Conference Series, ACM Siggraph, Addison-Wesley, Longman, (1999), 83-90.

[18] Y. Wang, J. Yang, W. Ying, Y. Zhang, A new alternating minimization algorithm for total variation image reconstruction, SIAM J. Imaging Sci., 1(2) (2008), 248-272.

[19] Z. Wang, A.C. Bovik, H.R. Sheikh, E.P. Simoncelli, Image quality assessment: from error visibility to structural similarity, IEEE Transactions on Image Processing, 13(4) (2004), 600-612.

[20] G. Wei, Generalized Perona-Malik equation for image processing, IEEE Signal Process. Lett., 6(7) (1999), 165-167.

[21] Y. You, M. Kaveh, Fourth-order partial differential equations for noise removal, IEEE Trans. Image Process., 9(10) (2000), 1723-1730. 